\title{
EchoGéo
}

$28 \mid 2014$

Police : les espaces de l'ordre, l'ordre en espace

\section{Espaces de l'insécurité : réalités territoriales et réponses locales à la délinquance}

Le cas de la région Midi-Pyrénées

Igor Lefèvre

\section{OpenEdition}

Journals

Édition électronique

URL : https://journals.openedition.org/echogeo/13836

DOI : 10.4000/echogeo.13836

ISSN : 1963-1197

Éditeur

Pôle de recherche pour l'organisation et la diffusion de l'information géographique (CNRS UMR 8586)

Référence électronique

Igor Lefèvre, "Espaces de l'insécurité : réalités territoriales et réponses locales à la délinquance », EchoGéo [En ligne], 28| 2014, mis en ligne le 08 juillet 2014, consulté le 21 septembre 2021. URL http://journals.openedition.org/echogeo/13836 ; DOI : https://doi.org/10.4000/echogeo.13836

Ce document a été généré automatiquement le 21 septembre 2021.

EchoGéo est mis à disposition selon les termes de la licence Creative Commons Attribution - Pas d'Utilisation Commerciale - Pas de Modification 4.0 International (CC BY-NC-ND) 


\section{Espaces de l'insécurité : réalités territoriales et réponses locales à la délinquance}

Le cas de la région Midi-Pyrénées

Igor Lefèvre

1 Interroger les problématiques de délinquance et de sécurité en les croisant avec les concepts de ruralité, de rurbanisation ou de périurbanisation ${ }^{1}$ reste relativement nouveau. En effet, les objets traditionnellement investis par la recherche en sciences sociales sur ces territoires portent sur l'environnement et les ressources naturelles, les mutations du monde agricole (Kayser, Schektman-Labry, 1982), ses modes d'organisation (Laborie, 1983) et de production d'espace (Jaillet, Jalabert, 1982), ou encore sur l'impact des mutations territoriales sur la fonction d'élu en milieu rural (Bruneau, Renahi, 2012). Un nombre restreint d'auteurs et d'analystes a développé une appréhension approfondie des territoires ruraux et périurbains dans le domaine de l'insécurité. En matière d'institution policière, il est possible de s'appuyer sur les travaux de François Dieu qui, à travers l'analyse du travail des militaires de la gendarmerie ${ }^{2}$, a pu investiguer les spécificités et les problématiques des zones rurales et périurbaines. En ce qui concerne les villes moyennes, en revanche, les recherches sur l'insécurité et les politiques locales de prévention de la délinquance ont été plus largement développées ${ }^{3}$, avec des focales plus prégnantes sur la question des banlieues et plus largement de la gouvernance.

2 Cependant, au niveau local, plusieurs recherches ont émergé, depuis la fin des années 1990, volontairement orientées sur les villes moyennes et leurs territoires environnants, qu'ils soient ruraux ou périurbains avec l'objectif implicite ou explicite d'investiguer des territoires autres que métropolitains. Les enseignements recueillis lors de différentes enquêtes menées entre 1999 et 2009 par le Centre d'Etudes et de Recherches sur la Police (CERP) ${ }^{4}$, ont fait apparaître clairement que chaque territoire d'investigation présente, malgré des tendances communes plus larges, une histoire et une configuration singulières, qui l'inscrivent dans une dynamique territoriale plus 
complexe que ce que laissent entendre les catégories et définitions sociodémographiques.

Parmi les éléments de qualification qui ont pu émerger des terrains de recherche, le premier qu'il convient de souligner correspond au lien entretenu entre la métropole toulousaine et les zones satellites constituées par les villes moyennes et leurs territoires environnants, dans les départements limitrophes à la Haute-Garonne. En illustration, on peut citer les conclusions du rapport régional des Assises des territoires ruraux qui rappellent que l'augmentation de la population régionale s'observe surtout à partir de deux phénomènes : "en couronne autour des villes " et "en étoile autour de Toulouse, le long des axes de communication $»^{5}$. La proximité et l'accessibilité de ce centre métropolitain ont donc profondément modifié certains des territoires étudiés, comme l'illustre la carte ci-après (illustration 1) tant en termes d'urbanisation que de population. Ainsi en est-il avec la densification des échanges autoroutiers du sud du Tarn-et-Garonne et de l'ouest du Tarn par exemple. A contrario les territoires plus enclavés ou moins bien desservis comme les agglomérations de Castres et d'Auch par exemple, ont été préservés ou en ont souffert (selon le point de vue adopté), dans leurs développements socioéconomiques respectifs.

Illustration 1 - Distribution des communes de Midi-Pyrénées en fonction de leur temps d'accès à l'échangeur autoroutier le plus proche

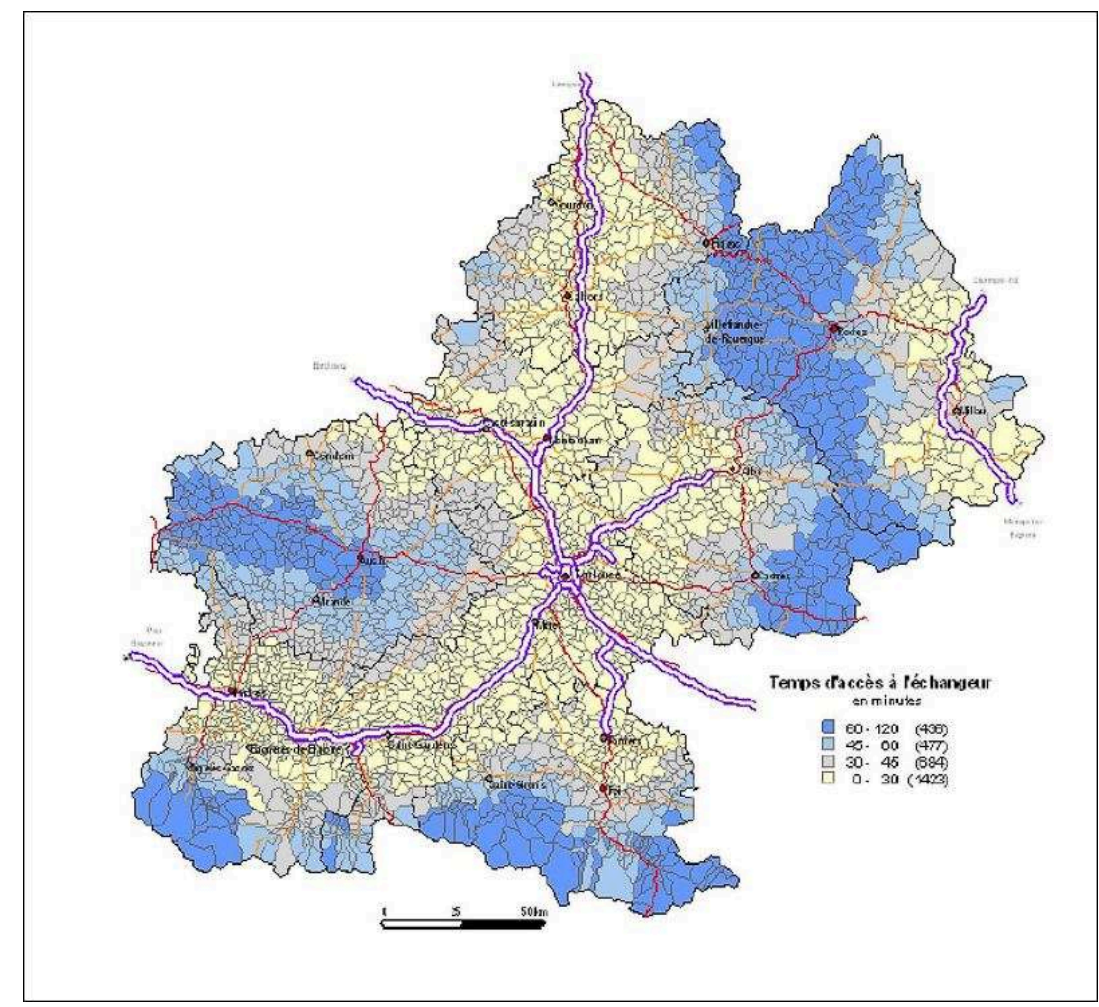

Sources : DDE 81 ; DRE Midi-Pyrénées 2005.

4 Un autre élément fort, issu de ces investigations, tient dans le questionnement récurrent concernant le " paradigme résidentialiste » : il s'agit d'étudier les différentes variables impactant les formes et l'intensité de la délinquance constatée selon les territoires étudiés. La criminologie aborde de manière transversale la dimension spatiale de l'activité criminelle et délictuelle. Pour définir le «paradigme 
résidentialiste ", on peut aborder ce concept à partir du postulat émis par plusieurs chercheurs selon lequel les objets environnementaux et géographiques constituent des éléments importants d'explication de la nature des faits et de la pression criminelle sur un territoire. Maurice Cusson (1986) a élaboré ce raisonnement en invitant les chercheurs à la prise en considération de l'analyse stratégique et de la théorie des opportunités. Felson et Cohen, concepteurs de la "routine activity approach», ont développé l'idée en ajoutant que «la probabilité qu'un crime soit commis en un lieu et à un moment donné est fonction de la convergence spatio-temporelle de délinquants potentiels, de cibles intéressantes et de l'absence de gardien capable de les protéger " (Felson, Cohen, 1980). La cible devient intéressante aux yeux du délinquant potentiel en fonction de quatre dimensions : sa valeur (ce qui rend la cible désirable pour le délinquant); sa visibilité, c'est-à-dire tout ce qui facilite la découverte de la cible par le délinquant potentiel ; son accessibilité, qui permet au délinquant d'entrer plus ou moins facilement en contact avec elle; et enfin son degré d'inertie, soit tout ce qui rend la cible plus ou moins difficile à emporter. Dès lors, on admet qu'existe une dichotomie entre rural et urbain concernant les types et volumes de délinquance constatée en fonction de ces éléments. La délinquance est plus importante et diversifiée en milieu urbain, du fait d'un nombre potentiel de victimes plus élevé, de cibles diversifiées et plus accessibles. Les opportunités criminelles y sont par ailleurs renforcées par la densité humaine plus forte et le desserrement progressif des liens et des modalités du contrôle social (Loubet-del-Bayle, 2012), qui ouvrent un champ d'action important aux délinquants potentiels. Ce constat impacte l'organisation de la réponse publique de sécurité avec d'un côté la concentration des forces de police nationale dans les villes pour faire face à une délinquance plus nombreuse et diversifiée, nécessitant de mobiliser rapidement des effectifs en cas de besoin et de l'autre, l'implantation de la gendarmerie nationale dans les territoires ruraux, afin d'assurer une surveillance générale et un maillage territorial optimisés, avec le ratio historique de 6 gendarmes par brigade territoriale compétente à l'échelle cantonale, pour prévenir et répondre à des formes spécifiques de délinquance rurale.

5 Au-delà de l'analyse des évolutions de la délinquance, les enquêtes résumées dans le présent article $^{6}$ ont permis d'observer les différences de niveau d'implication des acteurs locaux et les ressources qu'ils pouvaient mobiliser (institutionnelles, politicoadministratives, humaines, économiques, ou techniques) dans la mise en place des politiques publiques de sécurité et de prévention de la délinquance, dont une des caractéristiques est d'être élaborée "par le haut », en particulier par le ministère de l'Intérieur. Ces différences proviennent de trois variables repérées durant les investigations: 1) le niveau d'adéquation du champ territorial de compétence avec celui des problèmes à traiter, 2) la capacité à comprendre les problèmes de sécurité pour les traiter de façon adéquate et 3 ) leur inclinaison à intégrer un système local pour cela (Lefèvre, 2008).

6 Sur ces fondements, une problématique transversale s'est développée au gré des différentes recherches menées sur la région: quels impacts peuvent avoir les évolutions sociales et urbanistiques des territoires sur la sécurité (objective ou subjective) des habitants et dans quelles proportions influencent-elles les pouvoirs locaux dans les politiques publiques qu'ils mènent contre la délinquance? Sur cette base, le présent article s'attachera à mettre en perspective tout d'abord la transformation des problématiques de délinquance et d'insécurité liées aux évolutions 
des configurations géographiques et sociodémographiques des territoires puis l'impact que ces dernières peuvent avoir sur les réponses locales développées par les acteurs publics.

\section{L'impact de la transformation de l'organisation des territoires sur l'insécurité objective et le travail des forces de l'ordre}

7 Les résultats d'analyse des différentes investigations réalisées permettent de constater que la délinquance se déploie à partir des axes de communication, créant, autour de "bassins de vie sous influence urbaine», une pression plus importante que dans des zones plus enclavées. Ce principe ne s'applique pas pour les villes moyennes qui ont une criminalité locale déjà structurée et qui concentrent la majorité des faits constatés sur un territoire plus large (l'arrondissement ou le département), Ces analyses montrent que la délinquance constatée évolue en fonction du développement des réseaux de communication et de leur impact sur les territoires traversés ou à proximité. Ce qui n'est pas sans conséquence, au-delà des volumes, sur les types et formes de délinquance auxquels sont confrontés forces de l'ordre et habitants de ces territoires.

\section{Territoires de l'insécurité : l'enjeu des mobilités}

Dans les années 1960, l'explosion démographique consécutive au baby-boom, renforcée par l'exode rural, a surtout bénéficié aux pôles urbains. Dans la décennie suivante, un nouveau mode de croissance est apparu dans la plupart des villes françaises: la périurbanisation. Celle-ci procède à la fois, d'une concentration de l'emploi dans les pôles urbains, dont l'attractivité se trouve d'autant accrue, et d'un besoin d'espace de la part des familles, qui quittent les centres urbains saturés pour s'installer en périphérie, avec l'objectif de vivre dans un habitat pavillonnaire individuel. Ce phénomène a perduré au cours des années 1980 et 1990, partiellement compensé par des retours vers les pôles urbains. Conséquences de la périurbanisation, les déplacements quotidiens $\mathrm{du}$ domicile vers les lieux de travail, mais aussi vers les espaces de socialisation et de consommation, se sont considérablement amplifiés au fil des quarante dernières années.

Dès lors, cette diffusion des modes de vie et des valeurs de la société de consommation, associée à une amélioration des moyens de communication, réduisent singulièrement les frontières entre les mondes rural et urbain. Le secteur de la sécurité représente ainsi une illustration saisissante de ce nouveau continuum entre ruralité et urbanité, "la violence, la déviance et la peur ne connaissant guère les délimitations physiques et humaines, pas plus que les principes de répartition territoriale » (Dieu, 2005).

10 Ce constat amène alors à poser la question de l'échelon d'action territoriale le plus opératoire pour prévenir et lutter contre la délinquance? L'accroissement et l'accélération des mobilités physiques conduisent à concevoir le territoire, "non plus dans une logique de sédentarisation, mais de polarisation, d'attraction et de zone d'influence » (Dieu, 2005). Il en va de même pour la délinquance. Un même fait peut ainsi mettre en interaction trois types de territoires (celui de l'acte, celui de l'origine de la 
victime, celui de l'origine de l'auteur) qui peuvent présenter des différences importantes dans leurs dimensions géographiques, sociodémographiques et insécuritaires. Pour illustrer le propos, on peut prendre l'exemple d'un vol à l'arraché d'un téléphone portable, par un individu habitant en ville, au préjudice d'un autre habitant en zone périurbaine, sur le parking d'une zone commerciale périphérique. Cette situation va ainsi engager deux individus dans une relation de délinquance soudaine et imprévue, dans un espace qui n'est, pour aucun des deux, un lieu ordinaire de domiciliation et de vie. À travers cette interaction délictuelle, une multiplicité de territoires se croisent et interrogent la pertinence de la localisation des faits portés à la connaissance des forces de sécurité dans le cadre d'une politique de sécurité et de prévention. Est-ce celui du lieu du forfait, qui n'a été qu'un territoire d'opportunité ? Est-ce le lieu de vie du délinquant? Une fois le forfait commis, ce dernier y revient pour profiter du bien volé, mais aussi, peut-être, en réaliser le recel, ou en organiser la revente sur un marché parallèle, ce qui constitue d'autres infractions réalisées par le même auteur avec le même objet mais dans un autre lieu. Est-ce enfin le lieu de vie de la victime qui, une fois le traumatisme enduré, devra aller déposer plainte dans la brigade de gendarmerie de son canton, mais aussi attendre d'être informée par la Justice de l'évolution de son affaire, tout en ne pouvant bénéficier des dispositifs d'aide aux victimes, souvent centralisés dans les grandes agglomérations urbaines? Cet exemple montre qu'un fait délictuel peut recouvrir différentes dimensions territoriales, dont la prise en compte par les pouvoirs publics devrait permettre de représenter plus réellement les aspects des phénomènes délinquants et conduire à développer des réponses à partir de la notion de bassins de vie que portent les nouveaux territoires d'action publique, notamment les intercommunalités (Lefèvre, 2008).

\section{Formes et distribution de la délinquance constatée sur les territoires étudiés}

11 À partir des éléments formulés ci-avant, des variations, quant à la forme, à la périodicité et au volume de la délinquance constatée, peuvent dès lors se manifester selon des mouvements structurels ou conjoncturels.

L'augmentation du nombre d'habitants et le développement de l'activité économique dans les communes situées sur les trajets d'axes routiers et autoroutiers faisant le lien entre la métropole régionale et un chef-lieu de département transforment à la fois ces villes et villages dans leurs formes urbaines, leur démographie et leur sociologie, mais les rend surtout plus attractifs et accessibles pour certains types de délinquance. Par exemple, le croissant ouest tarnais irrigué par l'A $68^{7}$ reliant Toulouse à Albi, le sud du Tarn-et-Garonne avec l'A $62^{8}$ qui positionne Montauban sur le trajet entre les deux métropoles régionales Toulouse et Bordeaux, ou le sud-est du département du Gers qui, sous l'effet conjugué du développement du réseau routier et l'installation de plusieurs sous-traitants aéronautiques, a pu voir son urbanisation et sa population augmenter, à l'exemple de communes telles que Léguevin ou L'Isle-Jourdain.

Ces territoires - sur le constat des statistiques de la délinquance et des témoignages de nombreux acteurs (élus, agents des forces de l'ordre, associations locales, etc.) - sont devenus beaucoup plus attractifs qu'auparavant pour les délinquants locaux ou extérieurs. Ainsi, les villes et villages situés à proximité de ces axes routiers en plein développement ont subi des transformations de la criminalité constatée avec, en 
particulier, une augmentation des actes de délinquance acquisitive ${ }^{9}$. Les véhicules automobiles, les habitations et les produits à forte valeur ajoutée ou capacité de revente sur un marché parallèle (hifi, informatique, produits de beauté et vêtementsmaroquineries de luxe...), en constituent les principales cibles. Ce phénomène a été révélateur de deux tendances problématiques pour les acteurs de la sécurité publique. Tout d'abord, une présence plus marquée des délinquants "itinérants " par rapport aux délinquants locaux, avec un recours à la violence contre les personnes et les biens (dégradations, voiture béliers, etc...) plus systématique que les standards de la délinquance locale habituellement constatée. Les agents des forces de l'ordre interrogés mettent systématiquement en avant le nombre exponentiel d'affaires impliquant des «bandes toulousaines ou extérieures» profitant de la qualité et de l'accessibilité des réseaux routiers, pour préparer des «expéditions contre les lotissements ou les zones commerciales». Ensuite, cette délinquance importée rend encore plus difficile le travail d'enquête, car elle implique des délinquants peu ou pas identifiables rapidement par les forces publiques locales, les conduisant à être tributaires du degré d'implication que peuvent ou veulent bien avoir les autres services de police et de gendarmerie des territoires ou des départements voisins (le plus souvent la HauteGaronne), quand une identification est possible.

Pour illustrer ces mouvements structurels, on peut reprendre l'exemple du département du Tarn. Comme on peut le voir sur les cartes suivantes, la population a plus fortement augmenté, entre 1999 et 2005, le long du trajet de l'A 68 (de l'ouest du département vers le centre, c'est-à-dire de Lavaur à Albi en passant par le Gaillacois), ainsi que le long de la RN 126 qui relie la métropole Toulousaine vers le bassin de Castres-Mazamet (illustration 2). 
Illustration 2 - Variation (en \%) de la population par canton sur le département du Tarn entre 1999 et 2005

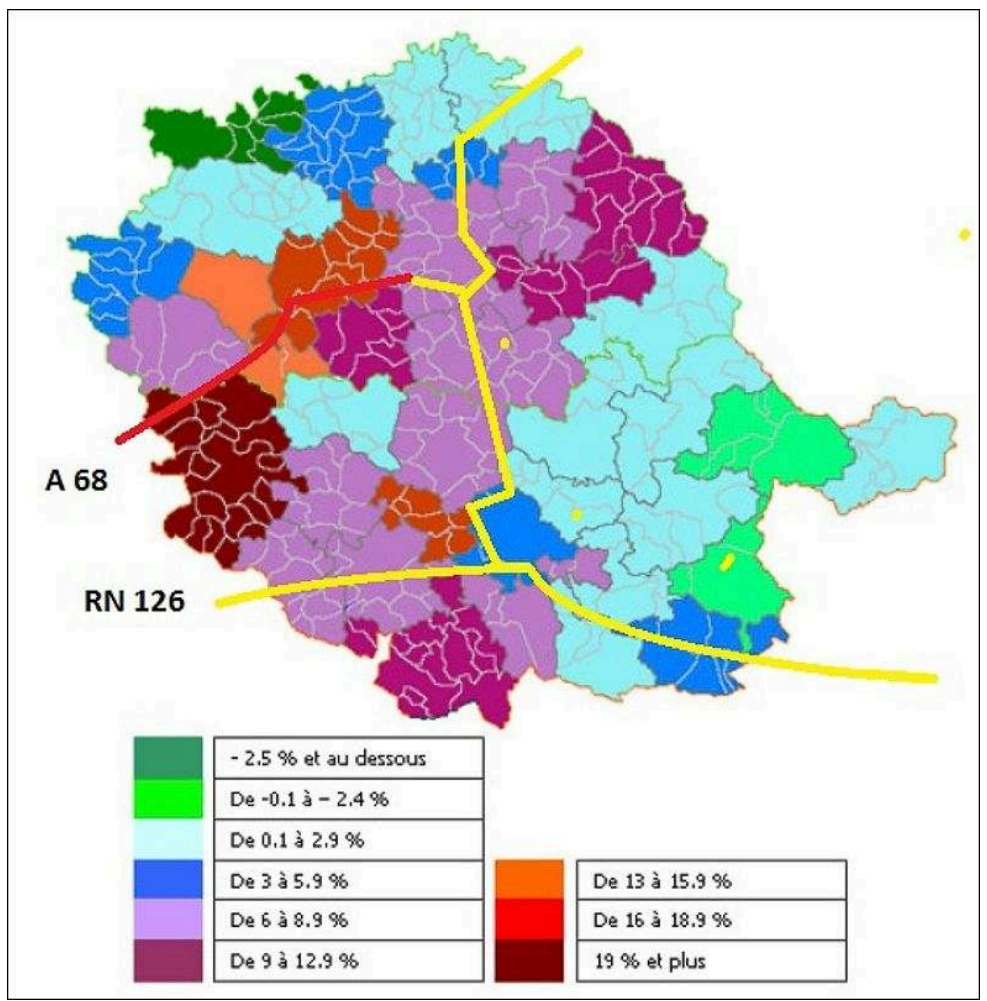

Sources : Estimations GN 81, DDSP 81 et données corrigées Insee.

Si l'on croise cette carte avec celle relative à la répartition communale des faits de délinquance constatée en 2005 (illustration 3), on remarque que les communes les plus touchées sont bien celles qui sont sur ces tracés routiers ou à proximité. Albi et Castres sont mises à part (en noir sur la carte) car leurs tailles respectives (50 et 45000 habitants) induisent des délinquances locales plus importantes que le reste du département. En effet, elles concentrent des situations et populations à risque que l'on ne retrouve pas dans le reste des communes du département. La tendance est encore plus remarquable à l'échelle des territoires de compétence des forces de l'ordre : ainsi entre 2001 et 2004 (illustration 4), les territoires en proximité des principaux tracés routiers du département subissent une augmentation de la délinquance constatée entre 3,9 et $34 \%$, selon les endroits, en particulier sur les territoires de compétence de la gendarmerie, calqués sur les regroupements de cantons (Dieu, 2004). 
Illustration 3 - Répartition communale de la délinquance générale constatée dans le département du Tarn en 2005 par les forces de l'ordre

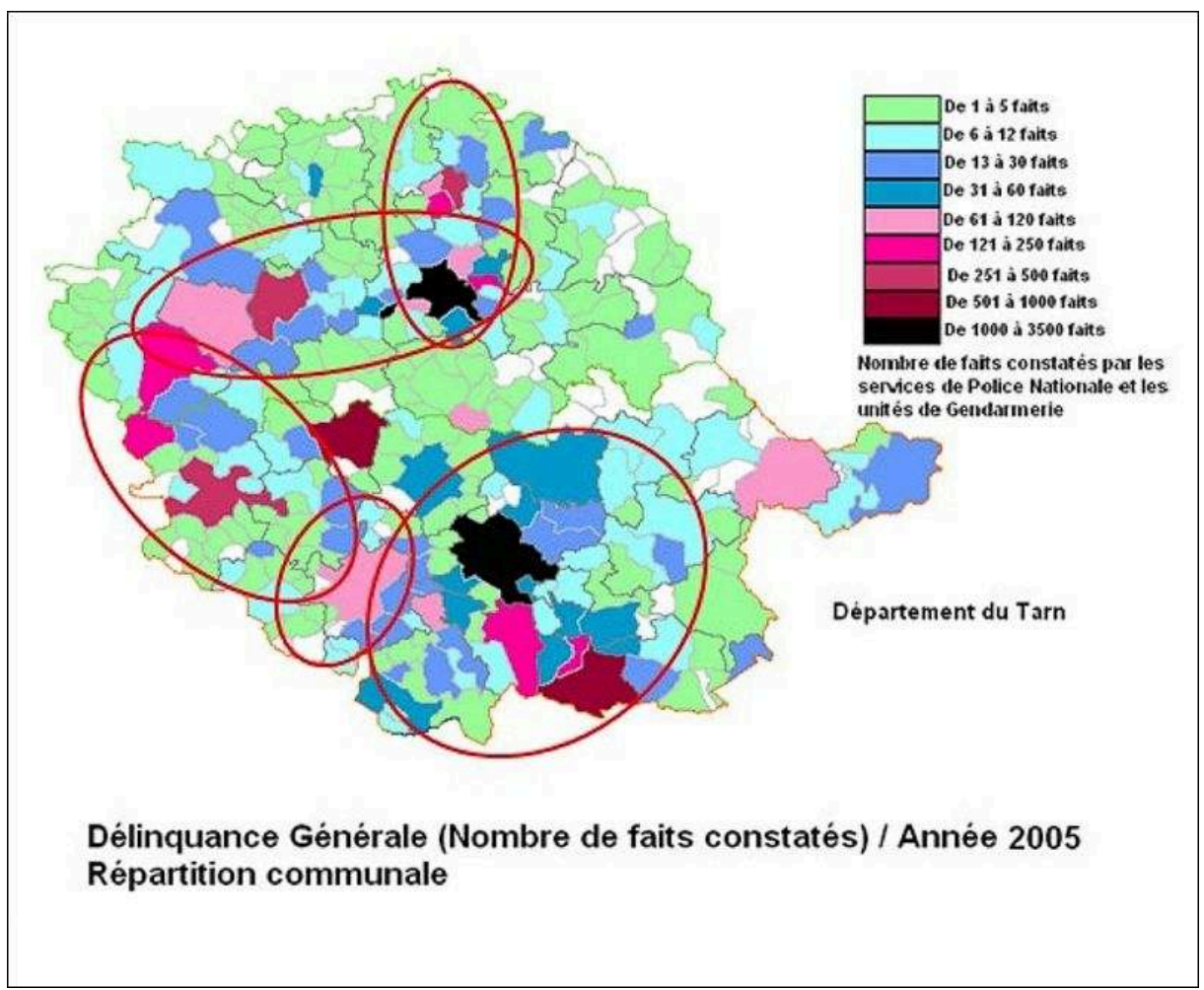

Sources : Groupement GN 81, DDSP 81.

Illustration 4 - Variation de la délinquance générale constatée dans le département du Tarn entre 2001 et 2004 par les forces de l'ordre

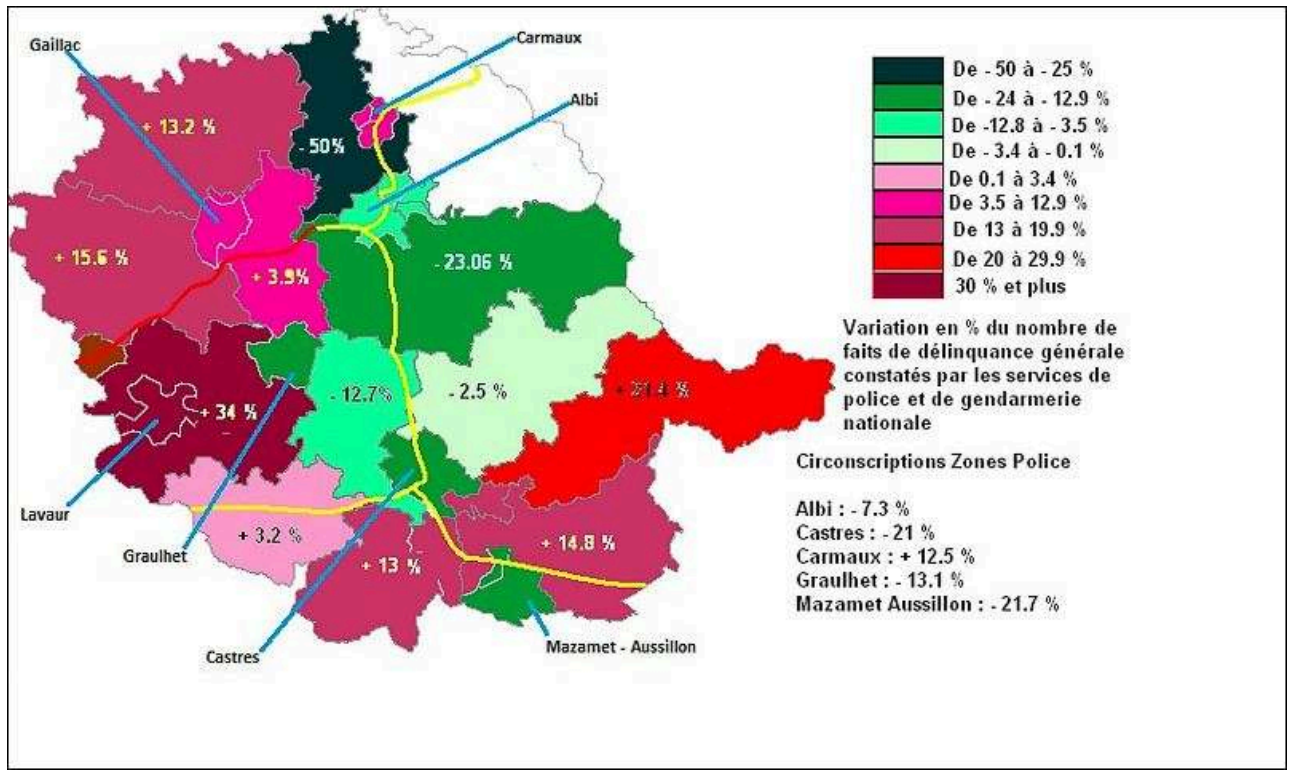

Sources : Groupement GN 81, DDSP 81.

16 En parallèle à ces mouvements structurels, des tendances conjoncturelles ont pu aussi être relevées. On peut en citer deux qui se retrouvent sur l'ensemble des territoires investigués. 
17 La région Midi-Pyrénées se caractérise par sa dimension touristique affirmée et une offre culturelle saisonnière relativement importante (expositions, festivals et événementiels d'envergure internationale). Dans la plupart des départements, comme le Gers, il a pu être constaté, par endroits, de très fortes variations temporelles quant à la répartition de la délinquance tout au long de l'année, avec notamment des pics entre juin et septembre, qui correspondent aux périodes d'afflux de populations liés à l'offre touristique importante (en particulier pour l'ouest du département). Dans d'autres cas, les rassemblements massifs festifs peuvent avoir un impact limité dans le temps, mais important sur les volumes d'actes de délinquance constatés par les agents de la force publique. En effet les risques pour l'ordre et la sécurité publique augmentent avec la concentration de populations diverses et nombreuses, sur un espace limité. C'est le cas notamment de communes telles que Marciac, Condom ou Gimont qui accueillent trois des plus importants événements festifs du département ${ }^{10}$, voire de la région, au printemps et en été. Les forces de l'ordre voient alors leur activité démultipliée et focalisée sur des faits spécifiques (la délinquance acquisitive avec les vols et pickpockets, les violences interpersonnelles et surtout de la consommation massive d'alcool par de nombreux protagonistes, l'ivresse au volant et l'émergence des infractions liées à la consommation et la détention de stupéfiants) ${ }^{11}$.

18 Les études de terrain montrent une deuxième tendance conjoncturelle, avec l'augmentation entre 2001 et 2006 des infractions liées à la consommation, la revente ou l'usage de stupéfiants dans l'ensemble des territoires investigués. Cette variation est due, en partie à la présence plus importante sur le terrain des forces de l'ordre, suite à la politique nationale d'abaissement de la tolérance des pouvoirs publics face à ces pratiques. Ce type d'infractions relève aussi de changements profonds dans la sociologie des publics jeunes de ces territoires. Comme ce fut analysé dans les enquêtes qualitatives sur les conduites addictives (CERP, 2007), l'ensemble des acteurs rencontrés (forces de l'ordre, professionnels de la santé et de la prévention) a pu constater un durcissement des pratiques à risque chez les mineurs et les jeunes majeurs. L'émergence, y compris dans les territoires ruraux, de polytoxicomanies, ainsi que l'apparition plus fréquente de drogues dures dans les saisies, s'expliquent, d'aprèseux, par l'intensification des échanges entre la métropole régionale et les territoires alentours. Cette situation facilite le contact entre consommateurs potentiels et produits disponibles. Dès lors, même si les volumes de consommation sont de moindre importance que dans les grandes villes et les villes moyennes, les habitants des territoires ruraux et périurbains ont désormais accès "à une offre de stupéfiants largement plus diversifiée qu'auparavant", ce qui tend vers "une homogénéisation des pratiques quel que soit le territoire d'analyse $»^{12}$.

19 Sur le fondement de ces éléments, rapidement résumés dans cet article, les recherches ont pu démontrer que le paradigme résidentialiste ${ }^{13} n^{\prime i n t e r v i e n t ~ q u e ~ p a r t i e l l e m e n t ~}$ dans l'explication de certaines formes de délinquance. Les territoires ruraux et périurbains, du fait de leurs évolutions sociodémographiques et économiques, mais aussi de leur accessibilité facilitée qui les rend de plus en plus poreux à l'urbain, ne paraissent plus aussi préservés que les représentations le laissaient penser.

20 En effet, si la diversité des territoires et leurs spécificités ont conduit à constater des différences certaines dans les volumes de faits constatés, pour autant, les tendances sur des périodes moyennes de cinq ans pointent vers une certaine homogénéisation dans les formes de la délinquance constatée. Malgré les différences de territoire, cela laisse 
entrevoir pour le futur une porosité renforcée entre délinquance urbaine et délinquance rurale. Ce rapprochement des formes de délinquance est lui-même tributaire de l'évolution des territoires, dans la façon dont ils sont habités, investis et utilisés par les habitants. De sorte qu'avec le phénomène de périurbanisation à l'échelle régionale et les transformations (urbanistiques, démographiques, sociologiques) qui en résultent, le paradigme résidentialiste s'avère être un outil, certes utile, mais qui gagnerait à être complété par d'autres critères, afin de proposer une lecture plus à même de décrire les réalités de l'insécurité dans des espaces impactés par le changement.

\section{Un sentiment d'insécurité exacerbé par le développement des problèmes liés au « vivre-ensemble »}

21 Au-delà de la question du rapprochement des formes de délinquances subies par les habitants dans les territoires urbains, périurbains ou ruraux, un autre thème a émergé de ces enquêtes de terrain : l'apparition de tensions concernant le "vivre-ensemble ", la cohésion sociale, dans des territoires et des communes soumis à de réels changements sociodémographiques. Le brassage des différentes générations d'habitants a parfois du mal à s'opérer, en particulier dans les zones périurbaines et rurales.

Plusieurs éléments communs à tous les départements investigués ont pu être repérés. On peut ainsi citer le cas des nuisances liées au bruit et à la promiscuité, en particulier dans les villes et villages qui ont connu un fort mouvement d'urbanisation et de peuplement au fur et à mesure que la métropole toulousaine s'est déployée à partir des années 1990. La difficile cohabitation entre plusieurs génération de populations ( «de souche " vs « nouveaux arrivants ») et la stigmatisation de certaines catégories d'entre elles sont des constantes dans l'observation. Ainsi, les adolescents dans les villages et petites villes sont perçus par les adultes et personnes âgées comme les principaux fauteurs de trouble à l'ordre public, les « nouveaux arrivants » dans les villes moyennes sont accusés par les habitants natifs ou anciens de dénaturer les représentations collectives traditionnelles de ces villes; ou enfin, les «étrangers » font des boucsémissaires idéals dans certains bassins de vie situés sur des axes de communication internationaux, dont l'activité économique repose sur des secteurs faisant appel à des mains d'œuvre précaires et saisonnières, comme par exemple le sud et l'ouest du Tarnet-Garonne avec l'agriculture maraîchère intensive.

Ces confrontations entre anciens et nouveaux habitants, mais aussi les représentations qu'elles suscitent, favorisent l'apparition d'un sentiment d'insécurité. Le travail d'enquête auprès des forces de l'ordre, mais aussi des élus locaux - souvent considérés par les habitants comme des tiers médiateurs permettant d'éviter le contact direct avec l'autre - a permis de comprendre les difficultés de régulation qui peuvent apparaître. En effet élus et fonctionnaires sont amenés à intervenir pour réguler ces situations de conflits latents générés par certains types d'incivilités. Le bruit et les occupations " abusives" d'espaces ou d'équipements publics constituent les générateurs les plus récurrents de ces situations de tension. Ainsi, les places, squares, parcs et arrêts de transports en commun fréquemment investis par les jeunes, représentent des points de fixation et de crispation dans les villes et villages où les infrastructures socioéducatives sont déficientes. Ces sites deviennent alors de véritables enjeux de conflit et 
l'intervention des acteurs locaux n'en est rendue que plus difficile. Elle révèle toute la difficulté des décideurs locaux à la fois pour ce qui relève de leurs capacités d'adapter leurs infrastructures à de nouveaux publics locaux (jeunes et nouveaux arrivants), tout en évitant de troubler les modes de vie des habitants anciens qui peuvent constituer des soutiens durables au système politique local.

Les enquêtes de terrain ont montré, bien souvent une distorsion entre les niveaux de délinquance constatée et le sentiment d'insécurité exacerbé dans certains quartiers ou territoires. Dans sa dimension subjective, l'insécurité reflète pour une part l'état psychologique du sujet, avec les angoisses et les peurs qu'il peut éprouver face à une personne, un groupe, une situation. Insécurités objective et subjective peuvent être corrélées (Roché, 1998). Ainsi, dans les zones rurales et périurbaines étudiées, le sentiment d'insécurité correspond plus à une construction sociale et médiatique fortement relayée dans la population, qu'à une réelle expérimentation de la délinquance. Étant, jusqu'alors, très peu concernées par la délinquance, les populations rurales et périurbaines se préoccupent davantage d'autres aspects de la vie sociale: accès aux services publics et aux infrastructures, gestion du voisinage, etc., ce qui les rend plus sensibles à la délinquance et à la tranquillité publique. Au contraire, l'expression de la peur dans les quartiers sensibles, en particulier dans les villes moyennes (Dieu, Lafontan, 2008), s'est révélée plus structurelle et davantage en lien avec une victimation ${ }^{14}$ plus importante, dont la réalité et le volume des faits exposaient les habitants à une crainte plus fondée.

L'étalement urbain de la métropole toulousaine, les phénomènes de périurbanisation et de rurbanisation sur les départements alentours, avec le développement des mobilités que cela a engendré, ont transformé de nombreux territoires, tant dans leur démographie que leur sociologie. Cela n'épargne pas les problématiques de sécurité notamment avec la mutation des formes de délinquance constatée - et les représentations contrastées selon les différentes catégories d'habitants. Ces transformations sont aussi remarquables dans leurs impacts quant à la production de sécurité par les acteurs publics, au premier rang desquels on peut trouver les forces de l'ordre, mais aussi sur l'organisation des réponses politico-administratives formalisées dans le partenariat entre l'État et les collectivités locales.

\section{Les réponses locales à la délinquance : entre difficultés d'action et transpositions hétérogènes de l'action publique}

Face aux évolutions conjuguées d'une délinquance plus mobile, de territoires plus difficiles à surveiller, et des modes de vie moins homogènes, la police et la gendarmerie ont dû adapter tant leur travail de surveillance que celui de police judiciaire. Mais ces changements ont aussi des conséquences sur les modes d'appréhension et de régulation des questions de sécurité par les systèmes politico-administratifs locaux. 


\section{Des conséquences sur l'organisation du travail et des missions des forces de l'ordre} d'une participation de plus en plus grande des polices municipales aux missions de surveillance, allant dans le sens d'une coproduction accrue de la sécurité. Ce partage des tâches a permis à la police nationale de se recentrer sur le travail de police judiciaire ou de pallier l'insuffisance des effectifs liée à la révision générale des politiques publiques (RGPP). Pour les territoires sous compétence de la gendarmerie nationale, cela s'est traduit par une modulation de l'activité de surveillance générale, avec une présence accrue dans les territoires les plus impactés par la délinquance, en délaissant ceux qui sont moins attractifs pour la délinquance (le rural profond et les territoires enclavés). Pour l'ensemble des gendarmes interrogés, les transformations urbanistiques et sociodémographiques de certains villages ou villes sous leur compétence ont impacté considérablement leur travail de surveillance et de contact avec la population. La généralisation d'un mode de vie fondé sur le navettage domiciletravail les conduit à surveiller des grandes poches vides d'habitants (lotissements, programmes immobiliers) sans forcément avoir de résultats en cas de problèmes (par exemple lors de vagues de cambriolages), du fait du peu d'information qu'ils peuvent recueillir auprès des habitants absents dans la journée. Cette disponibilité raréfiée des habitants, conjuguée à leur repli dans la sphère privée, parfois même accentuée par les formes d'habitat (multiplication des « gated-communities " (Sabatier, 2005), résidences sécurisées, en particulier autour de Toulouse, dans l'ouest tarnais et au sud de Montauban) les conduit à ne plus connaitre des pans entiers « de la population saine » de leurs territoires de compétence, alors même que cela constitue une des dimensions traditionnelles du travail du gendarme (Dieu, 1999).

Dès lors, même s'ils étaient moins touchés que la moyenne nationale, les territoires étudiés ont quand même dû expérimenter un changement des modes de l'action régalienne dans la capacité et les moyens des forces de l'ordre et des décideurs locaux à produire de la sécurité et de la tranquillité publiques. Ce changement s'est vu, par ailleurs, amplifié par deux autres facteurs. Le premier est institutionnel avec l'application, à partir de 2002, des principes du nouveau management public (NMP) dans la gestion territoriale des forces de l'ordre et le rapprochement de la police et de la gendarmerie nationale ${ }^{15}$ qui en a suivi, en ce qui concerne tant les règles statutaires que les modes de commandement et de travail. Le second est d'ordre politique, avec la montée en compétence des maires et leur possibilité de créer ou réactiver des polices municipales ${ }^{16}$. Ainsi, au moment des enquêtes de terrain, hormis le cas des villes moyennes qui bénéficiaient de services de police municipale organisés et étoffés (souvent une dizaine d'agents), de nombreuses communes et quelques intercommunalités lançaient des recrutements, souvent de un à trois agents, pour créer une réponse communale à l'insécurité. Ces embauches étaient la plupart du temps effectuées pour faire face à la demande sociale de sécurité des habitants, mais aussi pour pallier les carences supposées par la réorganisation territoriale de la gendarmerie nationale, avec le regroupement des brigades territoriales dans un nouveau dispositif: les communautés de brigades ${ }^{17}$. 


\section{Les politiques locales de sécurité et de prévention de la délinquance : une institutionnalisation contrariée}

L'organisation et le pilotage des politiques de sécurité urbaine ont connu des transformations radicales depuis un demi-siècle. Les vingt-cinq dernières années sont les témoins d'une accélération de ce processus, avec la consécration des élus locaux comme " pivots» de ces politiques publiques (Lefèvre, 2008). Les maires ont donc pris une nouvelle envergure face à des préfets qui, en mettant à leur disposition des outils d'action publique et des moyens financiers, leur ont permis d'assurer cette tâche, dans un double objectif de transversalité et d'efficacité de l'action publique.

Il ne faut pas, pour autant, surévaluer les effets de ce mouvement de coproduction de la sécurité. Concept totémique, le "partenariat " renvoie bien plus souvent à une rhétorique de l'action publique qu'à une réalité sur le terrain (Lefèvre, 2008, 2009). Plusieurs raisons expliquent cela. Tout d'abord, la très forte hétérogénéité des moyens développés, en particulier par les exécutifs locaux, souvent du fait de stratégies politiques peu claires, mais aussi le déficit de connaissances des problèmes ou des moyens d'action, qui impactent considérablement l'organisation et l'effectivité de ces dispositifs (Bousquet, Dieu, 2010). Par ailleurs, l'ancrage des cultures professionnelles et l'existence de stratégies personnelles de certains acteurs aux intérêts parfois divergents constituent des freins puissants à l'action collective (Lefèvre, 2008).

L'observation de situations à fort ancrage local a conduit à mettre en évidence qu'à côté des acteurs « traditionnels » (État, justice, collectivités locales), de « nouveaux » acteurs agissent à la frontière des actions publiques et privées (associations, cabinets d'études et de conseil), autant pour des missions en amont (conseil et d'expertise), qu'en aval (réalisation de prestations ou exécution de certaines missions en délégation de service public). La cohabitation et le partenariat restent pour autant tributaires de leurs capacités à coopérer, à trouver des consensus pour définir les problèmes et élaborer des réponses. Le poids des cultures professionnelles et la balkanisation organisationnelle s'avèrent les principaux obstacles à l'action locale (Lefèvre, 2009).

Plusieurs facteurs peuvent expliquer les très fortes disparités dans la production des politiques locales de sécurité et de prévention de la délinquance en fonction des caractéristiques territoriales des lieux d'investigation (rural - périurbain - villes moyennes). Dans les communes rurales, le déficit patent d'expertise en matière d'ingénierie -souvent par manque de moyens et d'intérêt - fait que la politique locale de sécurité n'existe pas, voire se résume à la collaboration avec la gendarmerie, ou dans le meilleur des cas, suit les actions d'un dispositif décidé à une échelle politicoadministrative plus élevée (intercommunalité). Dans le périurbain, le manque de moyens, les difficultés des autorités territoriales à investir cette thématique, là encore par désintérêt, méconnaissance, ou crainte d'un désengagement progressif de l'État dans ses missions régaliennes, constituent des freins à l'institutionnalisation d'une réelle politique de prévention et de sécurité urbaine. De plus, ces dispositifs exigent de nouvelles compétences et le recrutement de personnels qualifiés ou l'attribution de ces missions à des agents déjà en poste s'avère difficile. Dans ces communes en expansion mais aux moyens contraints, c'est l'échelon supracommunal qui prend bien souvent le relai des villes pour l'engagement de techniciens spécialisés. Ce transfert de compétence facilite l'équilibre politico-administratif local: les communes n'ont pas forcément envie ou les moyens d'intervenir dans le champ de la sécurité, les 
intercommunalités récupèrent une compétence, justifiant de leur légitimité d'action, tout en servant d'instance de mutualisation. Les villes moyennes enquêtées n'échappent pas à ces observations. Bien que les personnels recrutés aient, très souvent, un niveau de connaissances et de compétences professionnelles qui leur permettent d'investir ces missions, là encore le degré d'investissement et la volonté d'action des autorités territoriales influent fortement sur l'effectivité et les résultats des politiques locales de sécurité.

Dans ce contexte de difficile professionnalisation des acteurs de la sécurité dans les municipalités (sauf pour les polices municipales), les services préfectoraux et déconcentrés de l'État ont des difficultés pour pallier ces carences qu'ils ont, parfois encouragées en insistant sur la nécessité de développer des instances partenariales, dans des communes n'ayant pas forcément les ressources nécessaires pour animer ce type de dispositif (CERP, 2006, 2007). Paradoxalement, on a pu observer que les services préfectoraux se révélaient, eux-aussi, difficilement à même de jouer un rôle d'acteurressource face aux communes et intercommunalités. Les départements investigués étant relativement préservés (hors la Haute-Garonne), les questions de sécurité n'ont que très récemment émergées dans les agendas locaux des cadres préfectoraux, jusqu'alors plus centrés sur le développement économique et l'aménagement du territoire. Ce n'est qu'en raison des incitations ministérielles et de la réorganisation des services, avec l'obligation de mise en place des "pôles sécurité intérieure" dans chaque préfecture, qu'une prise en compte et une primo-acculturation sur le sujet s'est réalisée dans ces institutions. Cette situation d'adaptation à marche forcée est venue alimenter une certaine inertie et la croyance - chez de nombreux élus - en l'inefficacité des dispositifs, en réalité mal connus et utilisés, à la fois par leurs promoteurs et leurs supposés animateurs.

34 L'institutionnalisation des politiques locales de sécurité s'est donc avérée disparate et inégale sur l'ensemble des territoires observés, même si les maires ont inscrit la sécurité aux agendas municipaux, au moins en se saisissant de l'opportunité de développer des outils à l'image de leurs représentations sur le traitement de la délinquance (social vs répressif) et en fonction des moyens et opportunités à leur disposition. C'est ainsi que dans différentes communes, au moment des enquêtes, la mise en place de fonds spécifiques par les services de l'État (Fonds interministériel pour la prévention de la délinquance, Lefèvre, Dieu, 2010), a permis de suivre certaines orientations gouvernementales en faveur d'un modèle de lutte contre la délinquance et l'insécurité orienté vers les principes de prévention situationnelle. À ce titre, le développement de la vidéosurveillance, encouragé par l'État, (Lefèvre, 2006, 2008) est un indicateur d'un basculement des exécutifs locaux qui témoignent d'un certain éloignement du modèle traditionnel jusqu'alors en cours en France, fondé sur l'intervention sociale et la prévention primaire (Dieu, Bousquet, 2010). Cependant, l'expertise restreinte, les moyens limités et l'apprentissage contrarié des acteurs locaux sont de puissants freins à l'action publique et au partenariat local. Ce constat reste tout de même à nuancer dans les communes urbaines, où un mouvement de professionnalisation est en cours depuis la fin des années 1990 (Lefèvre, 2008). Pour conclure, notre recherche permet d'établir les éléments suivants :

Tout d'abord, la dichotomie rural-urbain, n'intervient que partiellement dans l'explication de certaines formes de délinquance, du fait de la diversité des territoires et de leurs spécificités. Au vu des transformations urbanistiques, démographiques, 
socioéconomiques qui impactent les territoires d'études, il semble que ce soit dorénavant plutôt la fonction du territoire (résidentielle, commerciale, etc.) et les modes de vie des habitants et usagers, qui déterminent les risques de victimation.

Par ailleurs, du fait de ces transformations sur un temps relativement court, le sentiment d'insécurité apparaît en filigrane dans la compréhension de certains phénomènes, comme l'intolérance face à l'apparition de modes de vie différents des usages traditionnels. Cela est aussi révélateur d'une intensification de la demande et de l'exigence sociale de sécurité, quel que soit le territoire observé, même si cela semble plus fort dans les espaces ruraux et périurbains avec l'arrivée de nouvelles populations aux standards de vie plus diversifiés, allant même jusqu'à révéler certains paradoxes. C'est, en effet, dans ces territoires, moins touchés par la délinquance, que la demande de sécurité est la plus intense, mais s'avère difficile à satisfaire pour les forces de l'ordre, car elles ont du mal à entrer en contact avec les habitants, dont les modes de vie s'organisent désormais autour d'une forte mobilité, mais aussi un fort repli sur la sphère privée et l'amenuisement des contacts lors du retour au domicile.

Enfin, ces situations impliquent une transformation du travail policier pour ne pas perdre en efficacité et interroge la pertinence de la répartition traditionnelle des forces de l'ordre sur le territoire. Par ailleurs, en ce qui concerne les politiques locales de sécurité, les investigations ont montré que les situations locales sont hétérogènes et les politiques de sécurité plutôt caractérisées par une faible institutionnalisation et la prééminence des relations informelles. Le rôle de l'État est donc à interroger, face au faible niveau de connaissance et d'implication des acteurs pour ce type de politique, même si cela est à nuancer dans le cas des villes moyennes. En effet, dans de nombreux cas, l'État a impulsé la création de dispositifs locaux de sécurité sans prendre en compte le niveau minime d'expertise dans les communes concernées. Cela a conduit à des situations de blocage avec les acteurs locaux et notamment les maires, censés animer ces dispositifs. Il reste alors que la promotion et l'implication d'une échelle territoriale peut débloquer ces situations, avec, en particulier, l'investissement des intercommunalités sur cette thématique. Cette opportunité présente le double avantage de promouvoir une approche d'intervention à l'échelle d'un bassin de vie élargi et la mise à disposition d'une ingénierie qui peut pallier les difficultés de certaines communes à investir ce champ, que ce soit par choix, par crainte ou par désintérêt des exécutifs municipaux.

\section{BIBLIOGRAPHIE}

Bruneau I. et Renahi N., 2012. Une petite bourgeoisie au pouvoir. Sur le renouvellement des élus en milieu rural. Actes de la recherche en sciences sociales, $n^{\circ}$ 191-192, p. 48-67.

CERP, octobre 2005. Diagnostic territorial de sécurité. Sécurité publique et sécurité routière dans les territoires périurbains du Tarn-et-Garonne. 
CERP, octobre 2006. Diagnostic départemental de sécurité du Tarn. Analyse territoriale et réponses locales à la délinquance.

CERP, 2007. Délinquance et conduites addictives dans le département du Gers.

CERP, 2007. Délinquance et conduites addictives dans le département du Tarn-et-Garonne.

CERP, mars 2008. Plan départemental de prévention de la délinquance dans le Tarn-et-Garonne. Document d'appui.

CERP, septembre 2008. Enquête relative aux Conseils locaux et intercommunaux de sécurité et de prévention de la délinquance du Gers.

CERP, février 2009. Conduites addictives et à risque - État des lieux et dispositifs préventifs dans les établissements scolaires du Gers. Rapport pour la Préfecture du Gers.

Cusson M., 1986. L'analyse stratégique et quelques développements récents en criminologie. Criminologie, vol. $19, \mathrm{n}^{\circ} 1$, p. 53-72.

Dieu F., Mignon P., 1999. La force publique au travail. Deux études sur les conditions de travail des policiers et des gendarmes. Paris, L'Harmattan, Sécurité et société, 272 p.

Dieu F., 1999. Politiques publiques de sécurité. Paris, L'Harmattan, Sécurité et société.

Dieu F., 2002. La Gendarmerie. Secrets d'un corps. Bruxelles, Complexe, Théorie politique.

Dieu F., 2004. Réformer l'offre de sécurité dans les territoires ruraux français. In Roche S., Réformer la police et la sécurité. Les nouvelles tendances en Europe et aux États-Unis, Paris, Odile Jacob.

Dieu F., 2005. Territoires, Partenariat, Sécurité. MODAT Repères, n 31, Toulouse, GIPCVAT 3.

Dieu F., 2005. Police de la route et gendarmerie. Paris L'Harmattan, Sécurité et société.

Dieu F., 2008. Sociologie de la gendarmerie. Paris, L'Harmattan.

Dieu F., Bousquet-Roux A., 2010. Regards sur l'évaluation de la prévention de la délinquance en France, Les cahiers de la sécurité, $\mathrm{n}^{\circ} 14$, INHESJ - La documentation française.

Dieu F., Lafontan C., 2008. Les violences urbaines dans les petites villes et les villes moyennes de MidiPyrénées, dans le cadre du dispositif de soutien à la recherche en sciences humaines et sociales de la Région Midi Pyrénées.

Dieu F., Mignon. P., 2001. Sécurité et proximité. La mission de surveillance générale de la Gendarmerie. Paris, L'Harmattan, Sécurité et société.

Dieu F., Lefevre I., 2010. La sécurité à l'heure des nouvelles technologies de contrôle : l'exemple de la vidéosurveillance en France. Les cahiers de la sécurité, $\mathrm{n}^{\circ}$ 11, INHESJ-La documentation française.

Felson M.. Cohen L.E, 1980. Human Ecology and Crime : A Routine Activity Approach, Human Ecology, vol. 8, p. 384-406.

Jaillet M.-C., Jalabert G., 1982. La production de l'espace urbain périphérique. Revue géographique des Pyrénées et du Sud-Ouest, t. 53, fasc. 1, p. 7-26.

Kayser B., Schektman-Labry G., 1982. La troisième couronne péri-urbaine ; une tentative d'identification. Revue géographique des Pyrénées et du Sud-Ouest, t. 53, fasc. 1, p. 27-34.

Laborie J.-P., 1983. Des banlieues partout ... en Midi-Pyrénées. Revue géographique des Pyrénées et du Sud-Ouest, t. 53, fasc. 1, p. 187-199. 
Lefèvre I., 2006. La vidéosurveillance urbaine : approche comparée entre France et Grande Bretagne. In Dieu F. (dir.), Questions de sécurité : sociétalisation des réponses, globalisation des menaces, Paris, L'Harmattan, p. 67-97.

Lefèvre I., 2008. La mise en œuvre des politiques locales de sécurité et de prévention de la délinquance. Thèse de doctorat es Science Politique, Université des sciences sociales de Toulouse I.

Lefèvre I., 2009. Les systèmes locaux d'action publique de sécurité. Les cahiers de la sécurité, $\mathrm{n}^{\circ} 8$, INHESJ - La documentation française.

Levèvre I., 2013. La commune : échelon naturel de la prévention de la délinquance et $\mathrm{du}$ phénomène criminel ? In Debove F., Reanaudie O., Sécurité intérieure, les nouveaux défis, Paris, Vuibert, p. 109-124.

Lefèvre I., Lafontan C., Bousquet A., 2008. La lutte contre les conduites addictives dans deux départements ruraux. Les Cahiers de la Sécurité, $\mathrm{n}^{\circ}$ 5, INHESJ - La documentation française, p. 88-95.

Loubet Del Bayle J.-L., 2012. De la police et du contrôle social. Paris, Éditions du Cerf.

Portet J.-P., 2005. Territoire et Sécurité, deux logiques opposées. Thèse pour le doctorat de science politique, réalisée sous la direction de F. Dieu, Université des Sciences Sociales de Toulouse 1.

Robert P., 1999. Le citoyen, le crime, l'État. Paris, Librairie Droz.

Roche S., 1998. Sociologie politique de l'insécurité : violences urbaines, inégalités et globalisation. Paris, Presses Universitaires de France.

Sabatier B. 2005. Les résidences fermées à Toulouse : une offre immobilière construisant la « ville privée ». Espaces et sociétés, 2005/4, p. 129-149.

\section{NOTES}

1. Pour les besoins de cet article, on entendra par périurbanisation, le phénomène d'urbanisation autour de la ville, rendant plus difficiles à tracer les limites avec l'espace rural. La périurbanisation se traduit généralement par un étalement urbain autour de l'agglomération. Le terme de rurbanisation, très proche, met davantage l'accent sur l'intégration d'un espace rural dans un ensemble urbain plus vaste. Cependant, en termes de conséquences pour les habitants et les usagers, si la périurbanisation se présente comme une extension du pôle urbain avec la vocation d'y être relié, la rurbanisation ne prétend pas offrir à ces derniers les facilités qu'on ne trouve habituellement pas à la campagne.

2. Voir la bibliographie à la fin du présent article pour une liste représentative des travaux écrits sur la question.

3. Les auteurs et les ouvrages sont trop nombreux pour que l'on puisse en faire le détail. Il convient néanmoins de se référer aux travaux de Jean-Jacques Gleizal, Sébastian Roché, Tanguy Le Goff, Jacques de Maillard, Jacques Donzelot, Philippe Robert et François Dieu.

4. Voir liste complète des recherches et études utilisées pour la réalisation de cet article dans la bibliographie de l'article.

5. Les «Assises des territoires ruraux » ont été initiées par le ministère de l'espace rural et de l'aménagement du territoire et conduites dans chaque département, d'octobre 2009 à janvier 2010, avec un pilotage préfectoral. Elles ont visé à identifier les atouts, les points faibles et les ressources de chaque territoire, à la lumière des observations réalisées par les professionnels et les habitants de ces zones. Certaines synthèses départementales et régionales se trouvent sur les 
sites Internet des préfectures ou des «Assises des territoires ruraux » : http://www.assisesterritoires-ruraux.gouv.fr/

6. Voir la liste des enquêtes dans la bibliographie. Concernant la méthodologie de travail, ces études se fondaient sur le croisement de plusieurs méthodes et outils des sciences sociales, avec l'utilisation des instruments d'analyse statistique pour mesurer les évolutions de la délinquance, l'observation de terrain et l'observation participante pour les enquêtes de terrain, et le recours aux méthodes de l'enquête vivante par l'usage systématique d'entretiens pour interroger les pratiques et représentations des acteurs locaux engagés dans la prévention de la délinquance et la sécurité urbaine.

7. CERP, octobre 2006. Diagnostic départemental de sécurité du Tarn. Analyse territoriale et réponses locales à la délinquance.

8. CERP, octobre 2005. Diagnostic territorial de sécurité. Sécurité publique et sécurité routière dans les territoires périurbains du Tarn-et-Garonne.

9. On entend par délinquance acquisitive, tout acte de soustraction frauduleuse sur la chose d'autrui ou lui-même, avec ou sans recours à la violence.

10. Festival Jazz'in Marciac, Fêtes de Gimont et Festival des bandas de Condom qui peuvent regrouper plusieurs milliers de personnes dans ces communes sur des temps très courts.

11. CERP, 2007. Délinquance et conduites addictives dans le département du Gers.

12. CERP, 2007, Délinquance et conduites addictives dans le département du Tarn-et-Garonne.

13. On entendra synthétiquement ce concept comme la dichotomie existant entre rural et urbain, pour ce qui concerne les distinctions de formes et d'intensité de la délinquance constatée sur ces différents territoires.

14. On entend par victimation le fait de subir une atteinte, matérielle, corporelle ou psychique, ainsi que d'en être conscient, La notion de victimisation renvoyant, quant à elle à la tendance à conférer aux victimes un statut social et une attention particulière

15. Plus précisément, la LOPS (loi d'orientation et de programmation pour la sécurité) de 1995, puis la LOPSI (loi d'orientation et de programmation pour la sécurité intérieure) de 2002, ont esquissé un redéploiement des forces de police et de gendarmerie, mais ces réorganisations n'ont eu lieu qu'à la marge sous le poids des résistances locales. Il a fallu attendre 2005 pour observer des réaménagements visibles de la carte policière, notamment par les réorganisations opérées dans les zones périurbaines, qui ont conduit, en reprenant les principes de la révision générale des politiques publiques (RGPP) initiée à partir de 2007, au rattachement de la gendarmerie nationale au ministère de l'Intérieur par la loi du 3 août 2009.

16. La loi n99-291 du 15 avril 1999 relative aux polices municipales, dite « la loi Chevènement » du nom du ministre de l'intérieur de l'époque qui a initié cette loi, a eu pour objet de réaménager l'organisation et les compétences de la police municipale. Outre le fait qu'elle redéfinit les conditions d'interventions et les moyens de la police municipale, elle modifie aussi le statut de ses agents et leurs modes de recrutement.

17. Concentration sur un même lieu des moyens et personnels de trois brigades territoriales ou plus, afin de rationaliser la présence et les moyens d'intervention des unités territoriales de la Gendarmerie. 


\title{
RÉSUMÉS
}

En prenant appui sur plusieurs études et recherches-actions menées par le Centre d'études et de recherches sur la police de l'Université Toulouse 1 Capitole (CERP) sur plusieurs territoires de la région Midi-Pyrénées, cet article s'attache à présenter sous quelles formes délinquance et insécurité objective se formalisent dans des bassins de vie, dorénavant impactés par la forte croissance de la métropole toulousaine. Il interroge ainsi la pertinence du paradigme résidentialiste, c'est-à-dire le postulat d'une délinquance dont les formes et l'intensité seraient largement tributaires des variables urbanistiques, sociologiques, économiques et démographiques selon les territoires investigués, ainsi que son impact sur l'organisation du travail et des missions des forces de l'ordre, mais aussi de l'ensemble des acteurs locaux engagés dans les politiques de sécurité et de prévention de la délinquance. À l'échelle de la région MidiPyrénées, cette contribution donnera à connaître synthétiquement quelles transformations des cadres et des modes de fonctionnement des politiques publiques ont pu émerger dans la décennie 2000-2010 dans des situations différentes, mais présentant toutes un élément commun: la nécessité d'agir face à des situations perçues par les acteurs locaux, comme risquées ou dangereuses pour la sécurité des habitants.

Based on several studies and research activities conducted by the Centre for Studies and Research on Police( CERP) from the University of Toulouse 1 Capitole, about several areas of Midi-Pyrénées, this paper seeks to present in which forms, crime and insecurity are formalized in living areas now impacted by the strong growth of the Toulouse metropolitan area. It thus questioned the relevance of the résidentialist paradigm (that is to say, the assumption of a crime would depend in form and intensity of urban, sociological, economic and demographic patterns and trends) and its impact on work organization and missions of law enforcement forces, but also all the local actors involved in security policy and crime prevention. At the level of MidiPyrénées, this contribution will give synthetically to know which transformations frameworks and patterns of public policies could emerge in the decade 2000-2010, in different situations, but all with a common element: the need to respond to situations perceived by local actors as risky or dangerous for the safety of citizens.

\section{INDEX}

Mots-clés : territoire, sécurité urbaine, délinquance rurale et urbaine, politique locale de sécurité, acteur public, acteur privé, ville-moyenne, rural, périurbain

\author{
AUTEUR \\ IGOR LEFÈVRE \\ Igor Lefèvre, I.LEFEVRE@ville-castres.fr, est attaché territorial, docteur en science politique, \\ chercheur associé au CERP (IEP/ UT1 Capitole).
}

